\title{
Visualization of the Magnetic Resonance Imaging Pulse Sequence
}

\author{
Zhiqiong Wang ${ }^{1, a}$, JunchangXin ${ }^{2, b}$, PeishunSun $^{1}$, liangboWang $^{1}$, ShuoTian $^{1}$ \\ ${ }^{1}$ Sino-Dutch Biomedical \& Information Engineering School,NortheasternUniversity, \\ Shenyang, 110004, China \\ ${ }^{2}$ College of Information Science \& Engineering, Northeastern University, \\ Shenyang, 110004, China

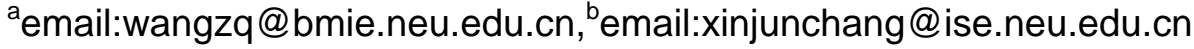

Keywords: MRI;Pulse sequence;Visualization;Graphical

\begin{abstract}
The pulse sequence is an important part of the magnetic resonance imaging (MRI). It is directly related to the resolution of medical image obtained by MRI, and other important parameters such as the imaging speed etc. Therefore, it is very important to develop newer and more efficient pulse sequence. This paper presents the visualization of the MRI pulse sequence. It can simplify the development process of new pulse sequence by designing the pulse sequence with graphicalmethod to replace the current mode of directly using text programming language to design it. Using the concept of a sequence of events and custom identifiers, we establish a middle interface between design environment of graphical MRI pulse sequence and text format programming environment recognized by nuclear magnetic resonance equipment.Itmakes the pulse sequence design open sources, and achieves independent to hardware and software. To avoid the tedious and complex programming process, the design of MRI pulse sequence is more efficient.
\end{abstract}

\section{Introduction}

With the development and popularization of MRI, we put forward higher requirements for the quality and imaging speed of the medical images obtained by magnetic resonance imaging. While the speed and image quality of MRI depend on the pulse sequence, the imaging process in MRI equipment of same magnetic field model, choosing an appropriate pulse sequence plays adecisive role in the imaging speed and image quality. The power of the magnetic resonance imaging technology is its flexibility that researchers can produce a variety of pulse sequences developed in different environments and applications to achieve the best scanning and imaging results. And the pulse sequence can be applied in many fields, and the using of pulse sequence is mentioned by many literatures [1][2].

In terms of the spectrometer, which is the core of magnetic resonance apparatus, at present a lot of spectrometers developed by laboratory [3][4]provide no pulse sequence development environment. References refer to the concept of digital nuclear magnetic resonance spectrometer [5]. Most of the pulse sequences designing environment of commercial nuclear magnetic resonance spectrometer use text programming environment. For example, the EPIC pulse sequence programming environment of the United States GE company uses the C language;The IDEA pulse sequence programming environment of the German SIEMENS company uses $\mathrm{C}++$; The sequence development platform provided by RI software of the British Resonance company uses Pascal language.

Although the pulse sequence design environment of text format is powerful and flexible control, there are still some shortcomings, such as requiring the users to have strong programming ability and to understand related knowledge of the pulse sequence design in-depth. Nowadays, with the development of computer technology and the popularization of magnetic resonance imaging equipment,the needs of simplified designing and humanity on the nuclear magnetic resonance (NMR) pulse sequences cannot be halted.As the graphical programming is simple, flexible and low threshold, some NMR simulation softwareprovides the graphical pulse sequence design 
environment [6][7], and some laboratory begin developing pulse sequence graphical design environment of pulse sequence [8], and there is a literature [9] referred to the design of graphical pulse sequence.The commercial spectrometers also provide graphical pulse sequence design environment, such as the NTNM of TECMAG Company, the SpinCad of Varian Company and the NMR-Sim of Bruker Company. However, at present most of these design environment are mainly for the development of NMR pulse sequence, although some also provide the design and development of MRI pulse sequence, in the design of complex MRI pulse sequence, its function has many limitations, and the pulse sequence design environment developed by each company can be only used in his own company's magneticresonance device.In orderto make the MRI pulse sequence design simplicity, humanization, visualization,we propose a visualization design of MRI pulse sequence.

\section{Material and Methods}

The design of the current mainstream MRI pulse sequence is written by the text format language, and then the magnetic resonance equipment implements the corresponding operation step by step orderly through recognizing the functions in program section. In this paper, the main idea is to realize the visualization of MRI pulse sequence design, using the sequence of events and custom identifier, establishing a middle interface between the graphical MRI pulse sequence design environment and the text format programming environment recognized by nuclear magnetic resonance equipment, to realize the free conversion between the graphical MRI pulse sequence and the programming language recognized by the nuclear magnetic resonance device.

\section{Design method}

\section{1). Event}

MRI pulse sequence actually meansthatin a period of time the nuclear magnetic resonance equipment performs continuously a series of concrete sequence operation process similar to apply a gradient field or RF pulse,etc. Thus, we used a concept called event. Every operation performed by MRI equipment is equivalent to an event, and the manifestation of the events in the pulse sequence isa cycle of MRI pulse sequencedivided into many small time quantums.In each time quantum,the state combination of $\mathrm{Rf}, \mathrm{Gz}, \mathrm{Gy}, \mathrm{Gx}$ in pulse sequence and parameter value information is an event.Among them, Rf stands for radio frequency (RF) pulse, Gz represents the level of selected gradient field, Gy represents the phase encoding gradient field, Gx represents the frequency encoding gradient field, in the same period of time the information of a group of Rf, Gz, Gy, Gx can be represented by an event. As shown in Figure 1, the MRI pulse sequence divided into a sequence of events, a simple gradient echo (GRE) sequence is divided into a set of events sequence composed of event.001 to event.014, for each event, the MRI equipment will perform a corresponding operation. For example, in Event.001, Rf, Gz, Gy, Gx are in the initial state, it means that nuclear magnetic resonance do not perform any operations and they are in the initial state. In Event.002, Rf, $\mathrm{Gy}, \mathrm{Gx}$ are in the initial state, but the $\mathrm{Gz}$ gradient field begins to increase and is on the rise, so Event.002 represents that the MRI equipment open level selection gradient fields and are in the level selection preparation stage. And so on, for all events the nuclear magnetic resonance equipment will execute the corresponding function, and have specific meaning.

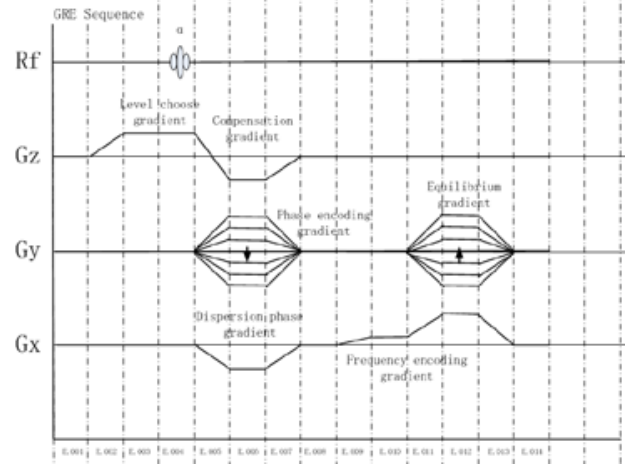

Fig.1.The MRI pulse sequence divided into a sequence of events 


\section{2). Identifier}

Creating a database and storing some custom identifiers in it, we call these custom identifiers which are defined by ourselves identifiers.Identifier is the text representation of events,mapping a corresponding independent event. For example,Subtracting a time period from pulse sequence, the events of this period is open and the RF pulse flip Angle is $90^{\circ}, \mathrm{Gz}$ gradient field to maintain a constant value, Gy gradient field and Gx gradient field intensity value is 0 .We can use the letter "A" to represent this event, "A" is equivalent to our custom identifier.Also, we can use the letter "B"to represent such an event that the value of $\mathrm{Rf}, \mathrm{Gz}, \mathrm{Gy}, \mathrm{Gx}$ is 0 , "B" is also our custom identifier.So on, if the "A", "B", "C", "D" are our custom identifier, and each letter of "A", "B", "C", "D" is only mapping one event,These four letters are mapping four kind of events, and each letter is mapping totally different events from others.We mark the possible events that exist in graphical map MRI pulse sequence with different custom identifiers. And store the mapping relation of each custom identifier with its corresponding events in a database table.And custom identifier is not only use a single letter "A", "B", "C", "D" to represent it, but using corresponding English acronyms of MRI equipment operation of each custom identifier as the name of custom identifier, using them to distinguish different custom identifiers.For example, custom identifier "Rlc" represents level selection stage,"SlcRF" represents that applying RF pulse to launch level selection stage.

\section{3). The MRI pulse sequence transforminto a sequence of events and its custom consist by identifiers}

Now, we have designed two kinds of available methods to implement the transition of graphic sequence of events which is divided by MRI pulse sequence into sequence of events which constitute custom identifier.

\section{Method 1:}

Let the custom identifier contains the state combination information of Rf, Gz, Gy, Gx and its parameter values.That is to say, a custom identifier contains all the information in an event.

Therefore, the state of a group of Rf, Gz, Gy, Gx combination in the same time period can be subdivided into more groups. The combination of Rf, Gz, Gy, Gx in each group is fixed. Besides, their values are sole. That is to say, the state combinations of different groups of Rf, Gz, Gy, Gx may be the same, while the value combinations are definitely different. We can subdivide the custom identifier, and increase a group of figures behind the custom identifier to distinguish the different combination of the value under the same state combination of Rf, Gz, Gy, Gx. For example, we use a custom identifier Rlc0100 to represent the state that the end of the level selection of the preparation phase and we can start the level selection, Gy, Gx are in the initial state, situate $\mathrm{Gz}$ a constant value of gradient field. Rlc represents the state, while 0100 represents a combination of a set of values. The same is true that the identifier Rlc02000's Rlc also represents this state, but 02000 represents combination of another set of values. In this way, each identifier is unique and can represent a class of Rf, Gz, Gy, Gx combination of state and value. Similarly, SlcRF represents that $\mathrm{Gz}$ gradient field continues to keep a constant value of the former events,applying RF pulse to launch level selection stage,and SlcRF90 represents the RF pulse flip Angle is $90^{\circ}$, SlcRF180 is on behalf of the RF pulse flip Angle is $180^{\circ}$.We choose "Rlc0100" "Rlc0200", "SlcRF90", "SlcRF180" directly as our custom identifier, then custom identifier contains all the information in an event. As shown in Figure 2 (a),Sketch Map of Methods:
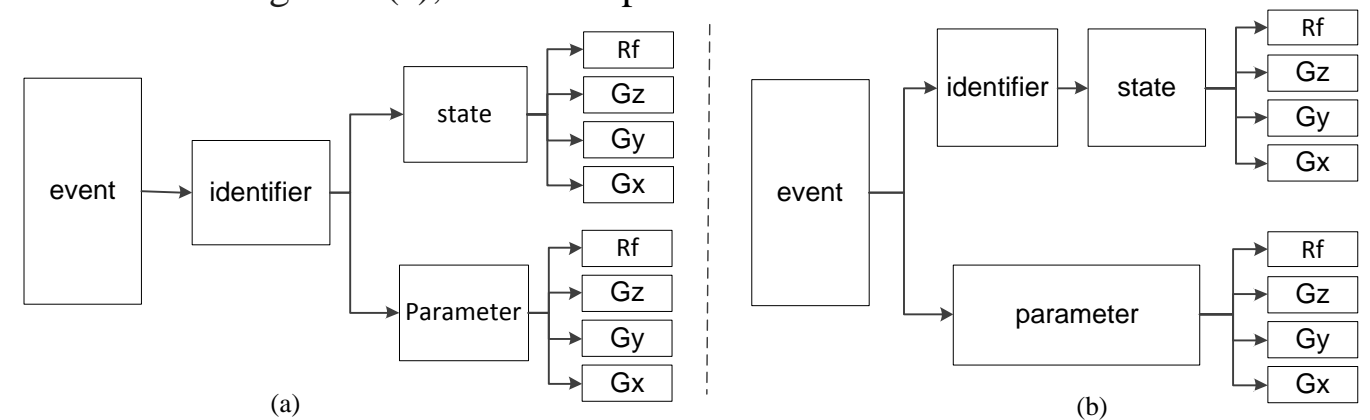

Fig.2.Sketch Map of Methods 
Then design special control component column in the visualization system which is developed by the Idea of visualization,each component in component column is a graphical representation of events, when we draw a MRI pulse sequence,select these components, automatically generate figure of each event in graphical MRI pulse sequence. In each event, the combination of state and values of Rf, Gz, Gy, Gx has one-to-one correspondence with customidentifier,

So the system can match each event an identifier in the database according to the state and input parameter values of Rf, Gz, Gy, Gx. Finally, the sequences of events divided by MRI pulse sequence can be transformed into a sequence of events which made up of identifiers.

\section{Method 2:}

Define the custom identifier represents state combination of Rf, Gz, Gy, Gx in an event while nothing relate with values. Set their values as parameter.Divide each event into two parts, the identifier and the parameters. That is, the custom identifier and the information in parameters that contains all the information in an event, the difference between method 2 and method 1 is that in Method 1 custom identifier contains all information of events,while in Method 2 the events information is divided into two parts, the state information and parameter values,custom identifier represents state information, and the parameters represent parameter value information.As shown in Figure 2 (b), Sketch Map of Methods.

After matching identifier for each event, the system assign values to the related properties of Rf, $\mathrm{Gz}, \mathrm{Gy}, \mathrm{Gx}$ according to the parameters we enter and make themstored in the parameters. Thus, the sequence of events divided by MRI pulse sequence can be converted into a sequence of events consisted by identifier and parameter.

Finally,implement the transition of sequence of events which is divided by MRI pulse sequence into sequence of events which constitute custom identifier and parameters,

\section{4).A sequence of events consisted by identifiers transfers into the language identified by MRI devices}

Although each company's MRI equipment underlying programming language is different, the process of programming allowing devices to perform operating effectively is similar. They are all from the respective programming environments calling the function library or operation code, each such function or a set of instructions can make MRI devices perform an operation. Meanwhile, a complete program or instruction set can make the MRI device execute a set of operations to realize the MRI pulse sequence. That is, a complete program or instruction set corresponds to a MRI pulse sequence. However, each identifiers in event sequence consisted by identifiers also corresponds to a operation preformed by MRI device. Thus, an identifier indirectly corresponds to a function section or a set of instructions identified by a MRI device. Therefore, it is easy for us to realize the conversion between identifier and the function section or group of instructions identified by MRI device, then the transformation between the event sequences consisted by identifiers and the language indentified by MRI devices can be realized. 


\section{Conclusions}

The method to realize the visualization of MRI pulse sequence by using identifiers provides a new idea. It can greatly improve the design efficiency of MRI pulse sequence. At the same time, it can make the design of MRI pulse sequence achieve the independence of hardware and software. It is an open method and can be turned into the language recognized by most of the MRI equipments only with slightly modification, and it has strong compatibility.

\section{Acknowledgement}

This research was partially supported by the National Natural Science Foundation of China under Grant Nos. 61402089, 61472069 and 61100022,the Fundamental Research Funds for the Central Universities under Grant Nos. N141904001, N130404014.

\section{References}

[1] C.R.Seuss, M. J.Kim, M. J.Triolo, C. H.Hajdu, and A. B.Rosenkrantz, ComparisonofMRI Pulse Sequences for Prediction of Size of Hepatocellular Carcinoma at Explant Evaluation, Am.J.Roentgenol.2014 (203), 300-305.

[2] Q.Gao, G.Srinivasan, R. L. Magin, and X. J. Zhou, Anomalous diffusion measured by a twice refocused spin echo pulse sequence: Analysis using fractional ordercalculus, J. Magn.Reson. Imaging. 2011 (33), 1177-1183

[3] S. M. Wright, D. G.Brown, J. R.Porter, D. C. Spence, E. Esparza, D. C.Cole, and F.R.Huson, A desktop magnetic resonance imaging system, Magn.Reson. Mater. Phys, Biol.Med. 2001 (13), 177-185

[4] P.Nicholas, D. Fushman, V. Ruchinsky, and D.Cowburn, The virtual NMR spectrometer: a computer program for efficient simulation of NMR experiments involving pulsed field gradients, J. Magn.Reson. 2000 (145), 262-275.

[5] L.Gengying, J.Yu, Y.Xiaolong, and J.Yun, Digital nuclear magnetic resonance spectrometer, Rev. Sci. Instrum. 2001 (72),4460-4463

[6] M.Helgstrand, P.Allard,QSim, a program for NMR simulations, J.Biomol.NMR. 2004 (30), $71-80$

[7] C.Cai, M.Lin, Z.Chen, X.Chen, S.Cai,andJ.Zhong, SPROM-an efficient program for NMR/MRI simulations of inter-and intra-molecular multiple quantum coherences, C.R.Phys. 2008 (9), 119-126 [8] S.Jie, L.Ying, L.Jianqi,andL.Gengying, A powerful graphical pulsesequence programming tool for magnetic resonance imaging, Magn.Reson.Mater.Phys., Biol. Med. 2005 (18), 332-342

[9] E.Mischler, F.Humbert, D.Canet, NMR pulse sequence generator for a PC driven spectrometer,Comput.Chem. 1998 (22), 219-223 